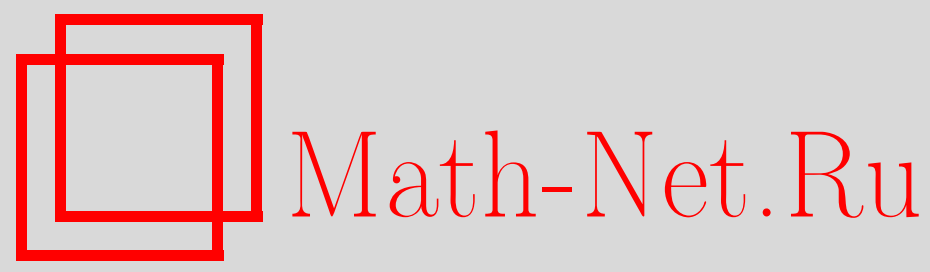

В. Ф. Кириченко, О геометрии подмногообразий Лагранжа, Матем. заметки, 2001, том 69, выпуск 1, 36-51

DOI: https://doi.org/10.4213/mzm482

Использование Общероссийского математического портала Math-Net.Ru подразумевает, что вы прочитали и согласны с пользовательским соглашением http://www. mathnet.ru/rus/agreement

Параметры загрузки:

IP : 3.89.197.203

26 апреля 2023 г., 08:17:26

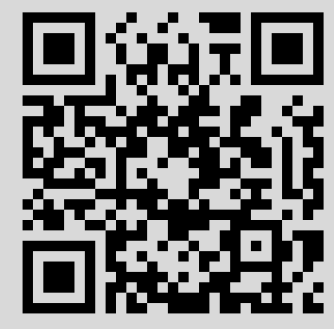




\title{
О ГЕОМЕТРИИ ПОДМНОГООБРАЗИЙ ЛАГРАНЖА
}

\author{
В. Ф. Кириченко
}

\begin{abstract}
Доказано, что через каждую точку произвольного симплектического многообразия в направлении любой лагранжевой плоскости проходит подмногообразие Лагранжа. Доказано, что подмногообразие Лагранжа с такими начальными данными, вообще говоря, не единственно, однако совокупность таких подмногообразий эрмитова расширения симплектического многообразия размерности свыше четырех при любых начальных данных содержит вполне геодезическое подмногообразие, названное нами $s$-лагранжевым подмногообразием, тогда и только тогда, когда это симплектическое многообразие является комплексной пространственной формой. Показано, что всякое подмногообразие Лагранжа комплексной пространственной формы голоморфной секционной кривизны $c$ является пространством постоянной кривизны $c / 4$. Найдены приложения этих результатов к геометрии главных тороидальных расслоений.

Библиография: 14 названий.
\end{abstract}

Подмногообразия Лагранжа, т.е. $n$-мерные подмногообразия $2 n$-мерного симплектического многообразия, аннулируюшие структурную форму, играют важную роль в симплектической геометрии и часто возникают в различных задачах механики и физики [1]-[3]. Это объясняется тем, что, с одной стороны, объекты симплектической геометрии часто изображаются лагранжевыми подмногообразиями. Например, график симплектоморфизма одного симплектического многообразия в другое является лагранжевым подмногообразием относительно естественной симплектической структуры в декартовом произведении этих многообразий [4]. С другой стороны, их значение в механике хорошо иллюстрируется тем фактом, что (полная) лагранжева поверхность, лежашая на уровне энергии $H(x, p)=E_{0}$ в фазовом пространстве механической системы, целиком содержит траектории гамильтоновой системы с гамильтонианом $H$, имеющие с этой поверхностью хотя бы одну общую точку [5]. Однако их геометрия подробно изучалась главным образом в пространстве $\mathbb{R}^{2 n}$, снабженном стандартной симплектической структурой. В настоящей работе изучаются подмногообразия Лагранжа в произвольном симплектическом пространстве $M$. Нами доказано, что через каждую точку $p \in M$ в направлении любой лагранжевой плоскости $L \subset T_{p}(M)$ проходит, вообще говоря не единственное, подмногообразие Лагранжа. С другой стороны, каждую симплектическую структуру на многообразии $M$ можно (неоднозначно) продолжить до почти келеровой структуры. Подмногообразия Лагранжа при этом являются не чем иным, как вполне вешественными подмногообразиями соответствующего почти келерова многообразия. Нами доказано, что если $\operatorname{dim} M>4$, то в классе вполне вещественных подмногообразий, проходящих через данную точку в данном вполне вещественном 
направлении, присутствует в точности одно вполне геодезическое подмногообразие, которое мы назьваем строго лагранжевым (короче, $s$-лагранжевым. ), тогда и только тогда, когда $M$ - комплексная пространственная форма. Доказано, что $s$-лагранжево подмногообразие комплексной пространственной формы (т.е. келерова многообразия постоянной голоморфной секционной кривизны $c$ ) является пространством постоянной кривизны $c / 4$. Рассмотрены контактные аналоги лагранжевых и $s$-лагранжевых подмногообразий. Контактньй аналог первых из них есть не что иное, как подмногообразия Лежандра контактного многообразия [3], а контактный аналог вторых назван нами подмногообразиями Бләра. Изучена связь между этими типами подмногообразий в случае, когда симплектическое многообразие $M$ имеет целочисленную структурную форму. В этом случае тотальное пространство главного $T^{1}$-расслоения над $M-$ расслоения Бутби-Вана - несет каноническую $K$-контактную структуру [6]. Нами доказано, что подмногообразия Лежандра относительно этой структуры есть не что иное, как горизонтальное поднятие лагранжевых подмногообразий, а подмногообразия Блэра - горизонтальное поднятие $s$-лагранжевых подмногообразий. Более того, мы доказьваем, что всякое $s$-лагранжево подмногообразие комплексной пространственной формы $M$ конечнолистно накрывается некоторым подмногообразием Блэра, а именно своим полным горизонтальньм поднятием в пространство расслоения Бутби-Вана над $M$.

Пусть $M$ - гладкое многообразие, $\mathrm{X}(M)-C^{\infty}(M)$-модуль гладких векторных полей на $M, d$-оператор внешнего дифференцирования. Все многообразия, тензорные поля и т.п. объекты предполагаются гладкими класса $C^{\infty}$. Напомним [1], что симплектической структурой на $M$ назьвается поле невырожденной замкнутой 2-формы, назьваемой структурной формой. Хорошо известно, что необходимыми условиями существования симплектической структуры на многообразии является его четномерность и ориентируемость. Многообразие, на котором фиксирована симплектическая структура, называется симплектическим многообразием. Если $p$-произвольная точка симплектического многообразия $M^{2 n}$, то $n$-мерное подпространство $L \subset T_{p}(M)$, аннулирующее структурную форму, называется лагранжевой плоскостью; $n$-мерноеподмногообразие симплектического многообразия $M^{2 n}$, касательное пространство которого в каждой точке является лагранжевой плоскостью, назьвается подмногообразием Лагранжса $[1]$.

Напомним [7], [8], что почти эрмитовой (короче, $A \mathscr{H}-$ ) структурой на многообразии $M$ назьвается пара $\{g, J\}$, где $g=\langle\cdot, \cdot\rangle$ - риманова метрика на $M, J$-почти комплексная структура, т.е. эндоморфизм касательного пучка многообразия $M$ такой, что $J^{2}=-$ id. При этом должно вьполняться тождество $\langle J X, J Y\rangle=\langle X, Y\rangle, X, Y \in \mathrm{X}(M)$. Из этих условий следует, что тензор $\Omega(X, Y)=\langle X, J Y\rangle$ кососимметричен; он называется фундаментальной формой структуры. Как и в случае симплектической структуры, необходимыми условиями существования $A \mathscr{H}$-структуры на многообразии является его четномерность и ориентируемость. Пусть $\nabla$ - риманова связность метрики $g$. Напомним [9], что почти эрмитова структура $\{g, J\}$ назьвается

- квазикелеровой $(Q \mathscr{K}-)$, если $\nabla_{X}(J) Y+\nabla_{J X}(J)(J Y)=0$,

- почти келеровой ( $A \mathscr{K}$-), если $d \Omega=0$,

- келеровой $(\mathscr{K}-)$, если $\nabla J=0$.

Хорошо известно [9], что $\mathscr{K} \subset A \mathscr{K} \subset Q \mathscr{K}$.

Пусть $M-2 n$-мерное почти эрмитово многообразие, т.е. многообразие, снабженное $A \mathscr{H}$-структурой; $n$-мерное подмногообразие $N \subset M$ назьвается вполне вещественным. если $\left.\Omega\right|_{N}=0$, т.е. $\langle X, J Y\rangle=0(X, Y \in \mathrm{X}(N))$. 
ОПРЕДЕЛЕНИЕ 1. Говорят, что почти эрмитово многообразие $M$ удовлетворяет аксиоме вполне вещественных подмногообразий, если в каждой точке $p \in M$ для каждого вполне вещественного подпространства $L \subset T_{p}(M)$ существует вполневещественное подмногообразие $N \subset M$, проходящее через точку $p$ и такое, что $L=T_{p}(N)$. Если каждое такое подмногообразие может быть выбрано вполне геодезическим, то говорят, что многообразие $M$ удовлетворяет аксиоме вполне вещественных плоскосmeй.

Симплектическая структура $\Omega$ всегда может быть (неоднозначно) дополнена до почти келеровой структуры, для которой форма $\Omega$ является фундаментальной формой структуры. В самом деле, известно [7], что при заданной невырожденной внешней 2-форме $\Omega$, исходя из произвольной римановой метрики $g_{0}$, всегда можно построить риманову метрику $g=\langle\cdot, \cdot\rangle$, переместимую с формой $\Omega$. Переместимость здесь означает, что эндоморфизм $J$ на $M$, однозначно определенньй тождеством $\langle X, J Y\rangle=\Omega(X, Y), X, Y \in \mathrm{X}(M)$, является почти комплексной структурой. Но тогда, как легко видеть, справедливо тождество $\langle J X, J Y\rangle=\langle X, Y\rangle, X, Y \in \mathrm{X}(M)$. Это означает, что пара $\{g, J\}$ образует почти эрмитову структуру на $M$, для которой форма $\Omega$ является фундаментальной формой структуры. Если $\Omega$ является симплектической структурой, т.е. замкнута, то соответствующая ей почти эрмитова структура является почти келеровой. Очевидно, такая структура строится неоднозначно (ввиду произвола в выборе метрики $g_{0}$ ). Будем называть ее эрмитовым продолжением исходной симплектической структуры.

ЗАмЕчАниЕ 1. Очевидно, лагранжевы плоскости симплектического многообразия являются не чем иным, как вполне вещественными подпространствами, а подмногообразия Лагранжа - вполне вещественными подмногообразиями любого эрмитова расширения этого симплектического многообразия.

Пусть $\mathscr{S}=\{g=\langle\cdot, \cdot\rangle, J\}-$ почти эрмитова структура на многообразии $M^{2 n}$, $p \in M$. Тогда в касательном пространстве $T_{p}(M)$ можно построить ортонормированный репер $\left(p, e_{1}, \ldots, e_{n}, J e_{1}, \ldots, J e_{n}\right)$. Такой репер будем назьвать вещественно адаптированным репером. С другой стороны, в комплексификации модуля $\mathrm{X}(M)$ внутренним образом определены два взаимно дополнительных проектора $\sigma=\frac{1}{2}(\mathrm{id}-\sqrt{-1} J)$ и $\bar{\sigma}=\frac{1}{2}(\mathrm{id}+\sqrt{-1} J)$ на собственные подмодули эндоморфизма $J$, отвечающие собственным значениям $\sqrt{-1}$ и $-\sqrt{-1}$ соответственно. Следовательно, можно построить репер $\left(p, \varepsilon_{1}, \ldots, \varepsilon_{n}, \varepsilon_{\hat{1}}, \ldots, \varepsilon_{\widehat{n}}\right)$ комплексификации пространства $T_{p}(M)$, где $\varepsilon_{a}=\sqrt{2} \sigma\left(e_{a}\right)$, $\varepsilon_{\widehat{a}}=\sqrt{2} \bar{\sigma}\left(e_{a}\right)$, состоящий из собственных векторов оператора $J_{p}$. Такой репер называется $A$-репером. Легко видеть, что матрицы компонент тензоров $J_{p}$ и $g_{p}$ в $A$-репере имеют вид соответственно

$$
\left(J_{j}^{i}\right)=\left(\begin{array}{cc}
\sqrt{-1} I_{n} & 0 \\
0 & -\sqrt{-1} I_{n}
\end{array}\right), \quad\left(g_{i j}\right)=\left(\begin{array}{cc}
0 & I_{n} \\
I_{n} & 0
\end{array}\right),
$$

где $I_{n}$ - единичная матрица порядка $n$. Хорошо известно [10], [11], что совокупность таких реперов определяет $G$-структуру на $M$ со структурной групой $U(n)$, представленной матрицами вида

$$
\left(\begin{array}{cc}
A & 0 \\
0 & \bar{A}
\end{array}\right)
$$

где $A \in U(n)$. Эта $G$-структура назьвается присоединенной. На протяжении всей работы будем подразумевать, что индексы $i, j, k, \ldots$ пробегают значения от 1 до $2 n$, 
индексы $a, b, c, d, f, g, \ldots-$ значения от 1 до $n$, и положим $\widehat{a}=a+n$. Поскольку $J$ и $g-$ тензоры типов $(1,1)$ и $(2,0)$ соответственно, их компоненты на пространстве расслоения всех комплексных реперов над $M$ удовлетворяют уравнениям

$$
\begin{aligned}
& \text { 1) } d J_{j}^{i}+J_{k}^{i} \omega_{j}^{k}-J_{j}^{k} \omega_{k}^{i}=J_{j, k}^{i} \omega^{k}, \\
& \text { 2) } d g_{i j}+g_{k j} \omega_{i}^{k}+g_{i k} \omega_{j}^{k}=g_{i j, k} \omega^{k}
\end{aligned}
$$

где $\left\{\omega^{i}\right\},\left\{\omega_{j}^{i}\right\}$ - компоненты форм смещения и римановой связности $\nabla$ соответственно, $J_{j, k}^{i}, g_{i j, k}$ - компоненты ковариантного дифференциала тензоров $J$ и $g$ в этой связности соответственно. Более того, в силу определения римановой связности $\nabla g=0$ и, значит,

$$
g_{i j, k}=0 \text {. }
$$

С учетом (1) и (3) соотношения (2) на пространстве присоединенной $G$-структуры перепишутся в форме

$$
\begin{aligned}
& \text { 1) } J_{b, k}^{a}=0, \quad \text { 2) } J_{\widehat{b}, k}^{\widehat{a}}=0, \\
& \begin{array}{ll}
\text { 3) } \omega_{\widehat{b}}^{a}=-\frac{1}{2} \sqrt{-1} J_{\widehat{b}, k}^{a} \omega^{k}, & \text { 4) } \omega_{b}^{\widehat{a}}=\frac{1}{2} \sqrt{-1} J_{b, k}^{\widehat{a}} \omega^{k}, \\
\text { 5) } \omega_{j}^{i}+\omega_{\hat{\imath}}^{\hat{j}} \equiv 0(\bmod n) .
\end{array}
\end{aligned}
$$

Кроме того, заметим, что в силу вешественности соответствующих форм и тензоров $\bar{\omega}^{i} \equiv \omega^{\hat{\imath}}(\bmod n), \bar{\omega}_{j}^{i} \equiv \omega_{\hat{\jmath}}^{\hat{\imath}}(\bmod n), \overline{\nabla J}_{j, k}^{i} \equiv \nabla J_{\hat{\jmath}, \hat{k}}^{\hat{\imath}}(\bmod n)$, где $t \rightarrow \bar{t}$ - оператор комплексного сопряжения. Несложно подсчитать, что почти эрмитова структура является квазикелеровой тогда и только тогда, когда на пространстве присоединенной $G$-структуры

$$
J_{\widehat{b}, c}^{a}=0
$$

При этом она является почти келеровой тогда и только тогда, когда

$$
\text { 1) } J_{\widehat{b}, c}^{a}=0, \quad \text { 2) } J^{[a b, c]}=0 \text {, }
$$

и является келеровой тогда и только тогда, когда

$$
\text { 1) } J^{a}{ }_{\widehat{b}, c}=0, \quad \text { 2) } J_{\widehat{b}, \widehat{c}}^{a}=0
$$

Здесь и в дальнейшем по индексам, заключенным в квадратные (соответственно круглые) скобки, подразумевается альтернирование (соответственно симметризация).

С учетом (5)-(7) нетрудно подсчитать, что первая группа структурных уравнений римановой связности

$$
d \omega^{i}=\omega_{j}^{i} \wedge \omega^{j}
$$

на пространстве присоединенной $G$-структуры $A \mathscr{H}$-многообразия запишется в следующей форме, называемой первой группой структурных уравнений АН̆Н-многообразия:

$$
\begin{aligned}
& d \omega^{a}=\omega_{b}^{a} \wedge \omega^{b}+B^{a b}{ }_{c} \omega^{c} \wedge \omega_{b}+B^{a b c} \omega_{b} \wedge \omega_{c}, \\
& d \omega_{a}=-\omega_{a}^{b} \wedge \omega_{b}+B_{a b}{ }^{c} \omega_{c} \wedge \omega^{b}+B_{a b c} \omega^{b} \wedge \omega^{c}
\end{aligned}
$$


где

$$
\begin{gathered}
\omega_{i}=g_{i j} \omega^{j}, \quad B_{c}^{a b}=-\frac{\sqrt{-1}}{2} J^{a}{ }_{\widehat{b}, c}, \quad B^{a b c}=\frac{\sqrt{-1}}{2} J_{[\widehat{b}, \widehat{c}]}^{a}, \\
B_{a b}{ }^{c}=\frac{\sqrt{-1}}{2} J^{\widehat{a}}{ }_{b, \widehat{c}}, \quad B_{a b c}=-\frac{\sqrt{-1}}{2} J^{\widehat{a}}{ }_{[b, c]} .
\end{gathered}
$$

При этом

$$
\begin{aligned}
& \text { 1) } \overline{\omega_{b}^{a}}=-\omega_{a}^{b}, \quad \text { 2) } \overline{B_{c}^{a b}}=B_{a b}{ }^{c}, \\
& \text { 4) } B_{c}^{(a b)}=0, \quad \text { 5) } \overline{B^{a b c}}=B_{a b c}
\end{aligned}
$$

Более того, с учетом (4) нетрудно получить, что

$$
J_{\widehat{b}, \widehat{c}}^{a}=4 \sqrt{-1} \widetilde{B}^{c a b}
$$

где $\widetilde{B}^{c a b}=\frac{1}{2}\left(B^{c a b}-B^{a b c}-B^{b c a}\right)$. Согласно (6) и (7) справедливо

ПРЕДЛОЖЕНИЕ 1. Почти эрмитова структура является квазикелеровой тогда и только тогда, когда $B^{a b}{ }_{c}=0$; является почти келеровой тогда и только тогда, когда $B_{c}^{a b}=0, B^{[a b c]}=0 ; u$ является келеровой тогда и только тогда, когда $B^{a b c}=0, B_{c}^{a b}=0$.

ЗАмЕчАниЕ 2. Очевидно, почти келеровость квазикелерова многообразия равносильна соотношению $B^{a b c}=\widetilde{B}^{a b c}$.

Пусть, в частности, $M$ - квазикелерово многообразие. Стандартная процедура дифференциального продолжения соотношений (9) с учетом предложения 1 приводит ко второй әруппе структурных уравнений квазикелеровой структуры:

$$
d \omega_{b}^{a}=\omega_{c}^{a} \wedge \omega_{b}^{c}+\left(2 B^{a d h} B_{h b c}+A_{b c}^{a d}\right) \omega^{c} \wedge \omega_{d}+B_{b c d}{ }^{a} \omega^{c} \wedge \omega^{d}-B_{b}^{a c d}{ }_{b} \omega_{c} \wedge \omega_{d}
$$

где $\left\{A_{b c}^{a d}\right\}$ - семейство функций на пространстве присоединенной $G$-структуры, служащих компонентами так называемого оператора кривизны присоединенной $Q$-алгебры [12], или структурного тензора второго рода, причем

$$
\text { 1) } A_{[b c]}^{a d}=0, \quad \text { 2) } A_{b c}^{[a d]}=2 B_{(b c) h} B^{h a d} .
$$

Кроме того,

$$
\begin{gathered}
d B^{a b c}-B^{h b c} \omega_{h}^{a}-B^{a h c} \omega_{h}^{b}-B^{a b h} \omega_{h}^{c}=B^{a b c}{ }_{h} \omega^{h}+B^{a b c h} \omega_{h}, \\
d B_{a b c}+B_{h b c} \omega_{a}^{h}+B_{a h c} \omega_{b}^{h}+B_{a b h} \omega_{c}^{h}=B_{a b c h} \omega^{h}+B_{a b c}{ }^{h} \omega_{h},
\end{gathered}
$$

где $B^{a b c}, B^{a b c d}, B_{a b c d}, B_{a b c}{ }^{d}$ - подходящие функции на пространстве присоединенной $G$-структуры. Отсюда, в частности, следует, что наборы функций $\left\{B^{a b c}\right\}$ и $\left\{B_{a b c}\right\}$ являются наборами компонент взаимно сопряженных тензоров на $M$, которые мы назовем структурными тензорами первого рода.

Сравнивая соотношения (12) со второй группой структурных уравнений римановой связности

$$
d \omega_{j}^{i}=\omega_{k}^{i} \wedge \omega_{j}^{k}+\frac{1}{2} R_{j k l}^{i} \omega^{k} \wedge \omega^{l}
$$


где $R^{i}{ }_{j k l}$ - компоненты тензора Римана-Кристоффеля, в силу линейной независимости базисных форм получим, что на пространстве присоединенной $G$-структуры

$$
\text { 1) } R_{\widehat{a} b c d}=2 B_{b c d}{ }^{a}, \quad \text { 2) } R_{\widehat{a} b c \widehat{d}}=2 B^{a d h} B_{h b c}-4 \widetilde{B}^{d a h} \widetilde{B}_{c b h}+A_{b c}^{a d} \text {. }
$$

Кроме того, с учетом (5), (6) и (11) имеем

$$
\text { 1) } \left.\omega^{a b} \equiv \omega_{\widehat{b}}^{a}=2 \widetilde{B}^{h a b} \omega_{h}, \quad 2\right) \omega_{a b} \equiv \omega_{b}^{\widehat{a}}=2 \widetilde{B}_{h a b} \omega^{h} \text {. }
$$

Дифференциальное продолжение этих соотношений с учетом (9), (14) и (15) приводит к равенствам

$$
\text { 1) } R_{a b c d}=4 \widetilde{B}_{[d|a b| c]}, \quad \text { 2) } R_{\widehat{a} \widehat{b} c d}=4 \widetilde{B}^{h a b} B_{h c d}
$$

плюс соотношения, полученные из (16) и (18) с учетом классических свойств симметрии и вещественности тензора Римана-Кристоффеля.

ОПРЕДЕЛЕНИЕ 2. Аभ̆-многообразие назьвается многообразием точечно постоянной голоморфной секционной кривизны с, если

$$
\forall X \in \mathscr{L} \Longrightarrow\langle R(X, J X) X, J X\rangle=c\|X\|^{4}
$$

Келерово многообразие точечно постоянной голоморфной секционной кривизны называется комплексной пространственной формой [8].

Пусть $M-A \mathscr{H}$-многообразие точечно постоянной голоморфной секционной кривизны $c$. Соотношение (19) на пространствеприсоединенной $G$-структуры запишется в фор-

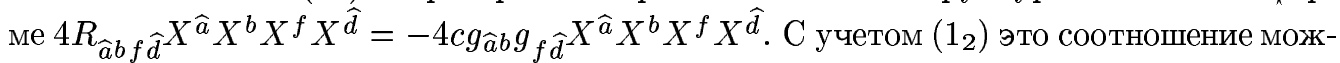
но переписать в виде

$$
\left(R_{\widehat{a} b f \widehat{d}}+c \delta_{b}^{a} \delta_{f}^{d}\right) X^{\widehat{a}} X^{b} X^{f} X^{\widehat{d}}=0
$$

Поляризация этого соотношения приводит нас к следующему результату.

ПРЕДЛОЖЕНИЕ 2. АН̈-многообразие является многообразием точечно постоянной голоморфной секционной кривизны с тогда и только тогда, когда

$$
R_{(b f)}^{(a)}=-\frac{c}{2} \widetilde{\delta}_{b f}^{a d}
$$

əде $\widetilde{\delta}_{b f}^{a d}=\delta_{b}^{a} \delta_{f}^{d}+\delta_{f}^{a} \delta_{b}^{d}$.

Пусть, в частности, $M$ - квазикелерово многообразие. Тогда с учетом $\left(16_{2}\right)$ соотношение (20) принимает вид

$$
A_{b f}^{(a d)}=4 \widetilde{B}^{(a d) h} \widetilde{B}_{(b f) h}-\frac{c}{2} \widetilde{\delta}_{b f}^{a d}
$$

Используя это соотношение и $\left(13_{2}\right)$, после упрощений получим 
ПРЕДЛОЖЕНИЕ 3. Квазикелерово многообразие является многообразием точечно постоянной голоморфной секиионной кривизны с тогда и только тогда, когда

$$
A_{b f}^{a d}=4 \widetilde{B}^{d a h} B_{(b f) h}-\frac{c}{2} \widetilde{\delta}_{b f}^{a d} .
$$

Пусть $M^{2 n}$ - квазикелерово многообразие, $N^{n} \subset M^{2 n}$ - вполне вещественное подмногообразие. Фиксируем точку $p \in N$. Пусть $\left(p, e_{1}, \ldots, e_{n}\right)$ - ортонормированный репер касательного пространства $T_{p}(N)$. Этот репер однозначно достраивается до вешественно-адаптированного репера $\left(p, e_{1}, \ldots, e_{n}, J e_{1}, \ldots, J e_{n}\right)$ касательного пространства $T_{p}(M)$. Пусть $\left(p, \theta^{1}, \ldots, \theta^{n}, \theta^{\hat{1}}, \ldots, \theta^{\widehat{n}}\right)$ - дуальньй корепер. Очевидно, сужение на $N$ семейства всех вешественно-адаптированных реперов многообразия $M$ определяет $G$-структуру со структурной грушой $U(n)$, в котором семейство $\mathscr{B}$ вешественно-адаптированных реперов указанного вьше вида образует подструктуру со структурной грушой $O(n, \mathbb{R})$, пространство которой задается системой Пфаффа

$$
\theta^{\widehat{a}}=0 .
$$

Нам, однако, удобнее будет иметь дело с главным расслоением $A$-реперов $\left(p, \varepsilon_{1}, \ldots, \varepsilon_{n}\right.$, $\left.\varepsilon_{\hat{1}}, \ldots, \varepsilon_{\widehat{n}}\right)$, порожденных реперами семейства $\mathscr{B}$. Напомним, что

$$
\varepsilon_{a}=\frac{1}{\sqrt{2}}\left(e_{a}-\sqrt{-1} J e_{a}\right), \quad \varepsilon_{\widehat{a}}=\frac{1}{\sqrt{2}}\left(e_{a}+\sqrt{-1} J e_{a}\right) .
$$

Поэтому матрища $C=\left(C_{j}^{i}\right)$ перехода от вещественно-адаптированного репера к соответствующему $A$-реперу имеет вид

$$
\left(C_{j}^{i}\right)=\frac{1}{\sqrt{2}}\left(\begin{array}{cc}
I_{n} & I_{n} \\
-\sqrt{-1} I_{n} & \sqrt{-1} I_{n}
\end{array}\right) .
$$

Следовательно, если $\left(p, \omega^{1}, \ldots, \omega^{n}, \omega^{\hat{1}}, \ldots, \omega^{\widehat{n}}\right)$ - корепер, дуальный $A$-реперу, то $\omega^{i}=$ $D_{j}^{i} \theta^{j}$, где $D=\left(D_{j}^{i}\right)$ - матрица, обратная матрице $C$. Очевидно,

$$
\left(D_{j}^{i}\right)=\frac{1}{\sqrt{2}}\left(\begin{array}{cc}
I_{n} & \sqrt{-1} I_{n} \\
I_{n} & -\sqrt{-1} I_{n}
\end{array}\right) .
$$

Следовательно,

$$
\text { 1) } \omega^{a}=\frac{1}{\sqrt{2}}\left(\theta^{a}+\sqrt{-1} \theta^{\widehat{a}}\right), \quad \text { 2) } \omega^{\widehat{a}}=\frac{1}{\sqrt{2}}\left(\theta^{a}-\sqrt{-1} \theta^{\widehat{a}}\right) .
$$

В частности, система Пфаффа (22) может быть переписана в форме

$$
\omega^{a}=\omega_{a} .
$$

Далее, первая группа структурных уравнений римановой связности на пространстве расслоения вещественно-адаптированных реперов многообразия $M$ имеет вид

$$
d \theta^{i}=\theta_{j}^{i} \wedge \theta^{j},
$$

где $\left\{\theta_{j}^{i}\right\}$ - компоненты формы римановой связности на этом пространстве. Поскольку $\theta^{i}=C_{j}^{i} \omega^{j}$, это соотношение можно также переписать в форме $C_{j}^{i} d \omega^{j}=\theta_{j}^{i} \wedge C_{k}^{j} \omega^{k}$ или

$$
d \omega^{i}=\left(D_{m}^{i} \theta_{k}^{m} C_{j}^{k}\right) \wedge \omega^{j} .
$$

С другой стороны, в силу (8) $d \omega^{i}=\omega_{j}^{i} \wedge \omega^{j}$. Сравнивая (28) с этим соотношением, получим, что $\left(D_{m}^{i} \theta_{k}^{m} C_{j}^{k}-\omega_{j}^{i}\right) \wedge \omega^{j}=0$, и в силу леммы Картана

$$
\omega_{j}^{i}=D_{m}^{i} \theta_{k}^{m} C_{j}^{k}+C_{j k}^{i} \omega^{k}
$$

где $\left\{C_{j k}^{i}\right\}$ - система функций на пространстве расслоения $A$-реперов, симметричная по нижним индексам. 
ЛЕмма. В принятых обозначениях $C_{j k}^{i}=0$.

ДокАЗАТЕльСтво. С учетом параллельности метрического тензора в римановой связности имеем

$$
\text { 1) } \left.d \widetilde{g}_{i j}+\widetilde{g}_{k j} \theta_{i}^{k}+\widetilde{g}_{i k} \theta_{j}^{k}=0, \quad 2\right) d g_{i j}+g_{k j} \omega_{i}^{k}+g_{i k} \omega_{j}^{k}=0,
$$

где $\left\{\widetilde{g}_{i j}\right\}$ и $\left\{g_{i j}\right\}$ - компоненты метрического тензора $g$ многообразия $M$ в вещественно-адаптированном репере и $A$-репере соответственно. Поскольку $g$ - тензор типа $(2,0)$, $g_{i j}=C_{i}^{k} C_{j}^{m} \widetilde{g}_{k m}$. С учетом этого $\left(30_{2}\right)$ примет вид

$$
\widetilde{g}_{m s} C_{k}^{m} C_{j}^{s} \omega_{i}^{k}+\widetilde{g}_{m s} C_{i}^{m} C_{k}^{s} \omega_{j}^{k}=0 .
$$

Подставляя сюда (29), после упрощений получим

$$
\widetilde{g}_{m s} C_{j}^{s} \theta_{t}^{m} C_{i}^{t}+\widetilde{g}_{m s} C_{i}^{m} \theta_{t}^{s} C_{j}^{t}+\left(\widetilde{g}_{k j} C_{i t}^{k}+\widetilde{g}_{i k} C_{j t}^{k}\right) \omega^{t}=0 .
$$

Но в силу $\left(30_{1}\right)$ сумма первых двух слагаемых суммы слева равна нулю, и в силу линейной независимости базисных форм $\widetilde{g}_{k j} C_{i t}^{k}+\widetilde{g}_{i k} C_{j t}^{k}=0$. Дважды делая в этом соотношении циклическую перестановку индексов $i, j$ и $t$, почленно сложим первые два соотношения и почленно вычтем третье: $\widetilde{g}_{k j} C_{i t}^{k}=0$. В силу невырожденности матрицы метрического тензора отсюда следует, что $C_{i t}^{k}=0$.

С учетом этой леммы соотношение (29) можно переписать в форме

$$
\theta_{j}^{i}=C_{m}^{i} \omega_{k}^{m} D_{j}^{k} .
$$

Выясним условия интегрируемости системы (22). В силу (27) условия $\left.d \theta^{\widehat{a}}\right|_{N}=0$ принимают вид $\theta_{b}^{\widehat{a}} \wedge \theta^{b}=0$, откуда в силу леммы Картана на подмногообразии $N$ должно выполняться соотношение

$$
\theta_{b}^{\widehat{a}}=H_{b c}^{\widehat{a}} \theta^{c},
$$

где $H_{b c}^{\widehat{a}}=H_{c b}^{\widehat{a}}-$ компоненты второй квадратичной формы погружения $N \subset M$. Заметим, что в силу (31)

$$
\theta_{b}^{\widehat{a}}=C_{h}^{\widehat{a}} \omega_{g}^{h} D_{b}^{g}+C_{\hat{h}}^{\widehat{a}} \omega_{g}^{\hat{h}} D_{b}^{g}+C_{h}^{\widehat{a}} \omega_{\hat{g}}^{h} D_{b}^{\hat{g}}+C_{\hat{h}}^{\widehat{a}} \omega_{\hat{g}}^{\hat{h}} D_{b}^{\hat{g}},
$$

откуда в силу (23) и (24) получаем

$$
\theta_{b}^{\widehat{a}}=\frac{\sqrt{-1}}{2}\left(-\omega_{b}^{a}+\omega_{b}^{\widehat{a}}-\omega_{\widehat{b}}^{a}+\omega_{\widehat{b}}^{\widehat{a}}\right) .
$$

В частности, (32) можно переписать в форме

$$
\omega_{b}^{a}-\omega_{b}^{\widehat{a}}+\omega_{\widehat{b}}^{a}-\omega_{\widehat{b}}^{\widehat{a}}=2 \sqrt{-1} H_{b c}^{\widehat{a}} \theta^{c} .
$$

Аналогично,

$$
\theta_{b}^{a}=\frac{1}{2}\left(\omega_{b}^{a}+\omega_{b}^{\widehat{a}}+\omega_{\widehat{b}}^{a}+\omega_{\widehat{b}}^{\widehat{a}}\right) .
$$

Заметим, что (33) можно записать в форме

$$
\left(\omega_{b}^{a}+\omega_{a}^{b}\right)+\left(\omega^{a b}-\omega_{a b}\right)=2 \sqrt{-1} H_{b c}^{\widehat{a}} \theta^{c} .
$$


Симметризуя и альтернируя это соотношение по индексам $a$ и $b$, получим соответственHо

$$
\text { 1) } \omega_{b}^{a}+\omega_{a}^{b}=2 \sqrt{-1} H_{(a b) c} \theta^{c}, \quad \text { 2) } \omega^{a b}-\omega_{a b}=2 \sqrt{-1} H_{[a b] c} \theta^{c},
$$

где $H_{a b c}=H_{b c}^{\widehat{a}}$. Переписав теперь (34) в форме

$$
\theta_{b}^{a}=\frac{1}{2}\left(\omega_{b}^{a}-\omega_{a}^{b}\right)+\frac{1}{2}\left(\omega^{a b}+\omega_{a b}\right)
$$

с учетом $(35),(17),(22)$ и (25) получаем

$$
\omega_{b}^{a}=\theta_{b}^{a}-\sqrt{2} \widetilde{B}^{c a b} \theta_{c}+\sqrt{-1} H_{a b c} \theta^{c} .
$$

ТЕОремА 1. Квазикелерово многообразие $M^{2 n}$ удовлетворяет аксиоме вполне вещественных подмногообразий тогда и только тогда, когда оно является почти келеровым многообразием.

ДокАЗАТЕЛЬСтво. Фиксируем точку $p_{0} \in M$ и $n$-мерное вполне вещественное подпространство $L \subset T_{p_{0}}(M)$. Пусть $N \subset M$-вполне вешественное подмногообразие, проходящее через эту точку в направлении $L$. Это означает, что $p_{0} \in N, T_{p_{0}}(N)=L$. Пусть $\left(p, e_{1}, \ldots, e_{n}\right)$ - ортонормированньй репер пространства $T_{p}(N)(p \in N),\left(p, \theta^{1}, \ldots, \theta^{n}\right)-$ дуальньй корепер. Как уже отмечалось, они порождают вещественно-адаптированный репер и дуальный ему корепер пространства $T_{p}(M)$ соответственно. Пусть $\left(p, \varepsilon_{1}, \ldots, \varepsilon_{n}\right.$, $\left.\varepsilon_{\hat{1}}, \ldots, \varepsilon_{\widehat{n}}\right)$ и $\left(p, \omega^{1}, \ldots, \omega^{n}, \omega_{1}, \ldots, \omega_{n}\right)$ - соответствующие им $A$-репер и дуальньй ему корепер. Согласно (22) подмногообразие $N \subset M$ задается системой Пфаффа $\theta^{\widehat{a}}=0$, и в силу (25)

$$
\text { 1) } \omega^{a}=\frac{1}{\sqrt{2}} \theta^{a}, \quad \text { 2) } \omega_{a}=\frac{1}{\sqrt{2}} \theta^{a} \text {, }
$$

(для простоты сужение формы на $N$ мы обозначаем той же буквой, что и саму форму). Систему (37) можно рассматривать как систему Пфаффа подмногообразия $N$. Найдем условия ее интегрируемости. Продифференцируем внешним образом $\left(37_{1}\right)$ :

$$
d \omega^{a}=\frac{1}{\sqrt{2}} d \theta^{a} .
$$

С учетом $(91),(27)$ и $(22)$ это соотношение примет вид

$$
\sqrt{2}\left(\omega_{b}^{a} \wedge \omega^{b}+B^{a b c} \omega_{b} \wedge \omega_{c}\right)=\theta_{b}^{a} \wedge \theta^{b}
$$

или в силу (37)

$$
\left(\omega_{b}^{a}-\theta_{b}^{a}\right) \wedge \theta^{b}+\frac{1}{\sqrt{2}} B^{a b c} \theta_{b} \wedge \theta_{c}=0 .
$$

Подставляя сюда (36), с учетом свойств симметрии второй квадратичной формы и линейной независимости базисных форм получим, что

$$
2 \widetilde{B}^{[b|a| c]}-B^{a b c}=0,
$$

что, очевидно, равносильно соотношению $B^{[a b c]}=0$ и в силу предложения 1 тому, что $M$ - почти келерово многообразие. Аналогично, с учетом $\left(9_{2}\right)$ и того обстоятельства, что в ортонормированном репере $\theta_{b}^{a}+\theta_{a}^{b}=0$, проверяется, что условие $d \omega_{a}=\frac{1}{\sqrt{2}} d \theta^{a}$ 
выполняется тождественно в силу (37). Таким образом, система (37) вполне интегрируема тогда и только тогда, когда $M$ - почти келерово многообразие. В этом и только этом случае через каждую точку $r$ пространства расслоения $A$-реперов, а значит, и пространства вещественно-адаптированных реперов, над $M$ проходит единственное интегральное многообразие максимальной размерности. Его проекция на $M$ является $n$-мерньм подмногообразием многообразия $M$, которое в силу (37) является вполне вещественньм подмногообразием, касательное пространство которого в начале вещественно-адаптированного репера $r$ совпадает с линейной оболочкой первых $n$ векторов этого репера. В силу произвола в выборе $r$ отсюда следует утверждение теоремы.

В качестве важного следствия доказанной теоремы получаем следующий результат.

СлЕдСТВИЕ. Через каждую точку р симплектического многообразия $M$ в направлении любой лагранжсевой плоскости $L \subset T_{p}(M)$ проходит подмногообразие Лагранжса.

ДокАЗАТЕЛЬСтво. В силу замечания 1 это утверждение равносильно справедливости аксиомы вполне вещественных подмногообразий для произвольного эрмитова расширения исходной симплектической структуры. Но в силу теоремы 1 эта аксиома справедлива, поскольку эрмитово расширение симплектической структуры, как уже отмечалось, является почти келеровой структурой.

ЗАмечАниЕ 3. Разумеется, этот факт следует также из классической теоремы Дарбу о локальной симплектоморфности симплектического многообразия стандартному симплектическому пространству. Наше рассуждение исходит из совершенно иных соображений.

ЗАмЕчАниЕ 4. Подмногообразие Лагранжа, проходящее через данную точку $p$ в направлении данной лагранжевой плоскости $L \subset T_{p}(M)$, вообще говоря, не единственно. Рассмотрим, например, стандартную симплектическую структуру на пространстве $\mathbb{R}^{2}$, заданную 2-формой $\Omega=d x^{1} \wedge d x^{2}$. Непосредственно проверяется, что подмногообразие $N_{1} \subset \mathbb{R}^{2}$, заданное уравнением $x^{2}=\left(x^{1}\right)^{2}$, равно как и подмногообразие $N_{2} \subset \mathbb{R}^{2}$, заданное уравнением $x^{2}=\left(x^{1}\right)^{3}$, являются одномерными подмногообразиями Лагранжа, проходящими через точку $O(0,0)$. Очевидно, их касательные пространства в точке $O$ совпадают и задаются уравнением $x^{2}=0$. Более обще, рассмотрим стандартную симплектическую структуру на пространстве $\mathbb{R}^{2 n}$, заданную 2-формой

$$
\Omega=\sum_{i=1}^{n} d x^{i} \wedge d x^{n+i} .
$$

Рассмотрим произвольную гладкую функцию $y=f\left(x^{1}, \ldots, x^{n}\right)$. Построим $n$ функций

$$
x^{n+i}=\frac{\partial f}{\partial x^{i}}, \quad i=1, \ldots, n .
$$

Рассмотрим $n$-мерное подмногообразие $N \subset \mathbb{R}^{2 n}$, заданное этой системой уравнений. Очевидно, оно является подмногообразием Лагранжа. Если фиксировать точку $p_{0}=$ $\left(x_{0}^{1}, \ldots, x_{0}^{n}\right) \in N$, то касательное пространство к $N$ в этой точке задается уравнением

$$
d x^{n+i}=\left.\frac{\partial f}{\partial x^{i} \partial x^{j}}\right|_{p=p_{0}} d x^{j}, \quad i=1, \ldots, n .
$$

$\mathrm{B}$ частности, если $f_{1}$ и $f_{2}$ - две функции, имеющие одинаковый гессиан в точке $p_{0}$, то они порождают, вообще говоря, различные подмногообразия Лагранжа, имеющие одинаковьй интегральный элемент в этой точке. 
ТЕОремА 2. Квазикелерово многообразие размерности свыше четырех удовлетворяет аксиоме вполне вещественных плоскостей тогда и только тогда, когда оно является комплексной пространственной формой и, следовательно, локально әквивалентно одному из следующих многообразий:

- при с>0 комплексному проективному пространству $\mathbb{C} P^{n}$;

- при с $=0$ комплексному евклидову пространству $\mathbb{C}^{n}$;

- при с $<0$ - комплексному гиперболическому пространству $\mathbb{C} H^{n}$.

ДокАЗАтЕльство. Необходимость немедленно вытекает из [11, теорема 38]. Обратно, пусть $M^{2 n}$ - комплексная пространственная форма. Надо доказать, что через каждую точку многообразия $M^{2 n}$ в любом $n$-мерном вполне вещественном направлении проходит единственное вполне геодезическое $n$-мерное интегральное многообразие. Заметим, что вполне геодезичность интегрального многообразия равносильна обращению в нуль его второй квадратичной формы [8], а потому требование вполне геодезичности интегральных многообразий системы (37) равносильно добавлению к этой системе уравнений (36) при $H_{a b c}=0$, которые мы запишем в форме

$$
\theta_{b}^{a}=\omega_{b}^{a} .
$$

Условие интегрируемости полученной системы Пфаффа задается соотношением

$$
d \theta_{b}^{a}=d \omega_{b}^{a}
$$

или с учетом (15)

$$
\theta_{c}^{a} \wedge \theta_{b}^{c}+\frac{1}{2} \widetilde{R}_{b c d}^{a} \theta^{c} \wedge \theta^{d}=\omega_{c}^{a} \wedge \omega_{b}^{c}+\omega_{\widehat{c}}^{a} \wedge \omega_{b}^{\widehat{c}}+\frac{1}{2} R_{b i j}^{a} \omega^{i} \wedge \omega^{j},
$$

где $\widetilde{R}_{b c d}^{a}$ - компоненты тензора Римана-Кристоффеля риманова многообразия $N$. Поскольку $M$ - келерово многообразие, $\omega_{b}^{\widehat{a}}=\omega_{\widehat{b}}^{a}=0$ в силу $\left(17_{1,2}\right)$. Кроме того, заметим, что ввиду вполне геодезичности подмногообразия $N \subset M$ имеем $\widetilde{R}=\left.R\right|_{N}$ и, значит,

$$
\widetilde{R}^{a}{ }_{b c d} \theta^{c} \wedge \theta^{d}=C_{i}^{a} D_{b}^{j} D_{c}^{k} D_{d}^{l} R_{j k l}^{i} \theta^{c} \wedge \theta^{d}=C_{i}^{a} D_{b}^{j} R_{j k l}^{i} \omega^{k} \wedge \omega^{l} .
$$

Подставляя эти соотношения в (40), после необходимых сокращений с учетом (17), (39) и линейной независимости базисных форм получим

$$
C_{i}^{a} D_{b}^{j} R_{j c d}^{i}=R_{b c d}^{a}+R_{b c \widehat{d}}^{a}+R_{b \widehat{c} d}^{a}+R_{b \widehat{c} \widehat{d}}^{a} .
$$

С учетом (23) и (24) это соотношение можно переписать в форме

$$
\begin{aligned}
& -R_{\widehat{a} b c d}-R_{\widehat{a} b c \widehat{d}}-R_{\widehat{a} b \widehat{c} d}-R_{a b \widehat{c} \widehat{d}}+R_{a b c d}+R_{a b c \widehat{d}}+R_{a b \widehat{c} d}+R_{a b \widehat{c} \widehat{d}} \\
& \quad+R_{\widehat{a} \widehat{b} c d}+R_{\widehat{a} \widehat{b} c \widehat{d}}+R_{\widehat{a} \widehat{b} \widehat{c} d}+R_{\widehat{a} \widehat{b} \widehat{c} \widehat{d}}+R_{a \widehat{b} c d}+R_{a \widehat{b} c \widehat{d}}+R_{a \widehat{b} \widehat{c} d}+R_{a \widehat{b} \widehat{c} \widehat{d}}=0 .
\end{aligned}
$$

С учетом (16) и (18) после необходимых сокращений окончательно получаем условия интегрируемости системы $(37),(39)$

$$
A_{b c}^{a d}-A_{b d}^{a c}+A_{a c}^{b d}-A_{a d}^{b c}=0 .
$$

Очевидно, на комплексной пространственной форме эти условия выполняются тождественно в силу предложений 1 и 3. Таким образом, система (37), (39) вполне интегрируема. Повторяя то же рассуждение, что и при окончании доказательства теоремы 1 , получаем, что через каждую точку $p$ многообразия $M$ в направлении любого вполне вещественного подпространства $L \subset T_{p}(M)$ проходит $n$-мерное вполне геодезическое вполне вещественное подмногообразие. 
ОпредЕлЕниЕ 3. Вполне геодезическое подмногообразие Лагранжа симплектического многообразия с фиксированным эрмитовым расширением назовем строго лагранжевым, короче, $s$-лагранжевым.

Из теоремы 2 немедленно следует

ТеОрема 3. Через каждую точку симплектического многообразия $M^{2 n}(n>2)$ с фиксированным эрмитовым расширением в направлении любой лагранжевой плоскости в этой точке проходит единственное $s$-лагранжево подмногообразие тогда и только тогда, когда это әрмитово расширение является комплексной пространственной формой.

ДокАЗАТЕЛьСтво. В силу замечания 1 утверждение теоремы 3 вытекает из справедливости аксиомы вполне вещественных плоскостей для эрмитова расширения исходной симплектической структуры. Единственность подмногообразия вытекает из его вполне геодезичности.

ЗАмЕчАнИЕ 5. Из доказательства теоремы 2 очевидно следует, что для комплексных пространственных форм утверждения теорем 2 и 3 справедливы без каких-либо ограничений на их размерность.

Пусть $N \subset M^{2 n}-s$-лагранжево подмногообразие почти келерова многообразия, $A \mathscr{K}$-структура которого рассматривается как эрмитово расширение симплектической структуры. В этом случае (36) запишется в форме

$$
\omega_{b}^{a}=\theta_{b}^{a}-\sqrt{2} B^{c a b} \theta_{c} .
$$

Запишем сужение второй группы структурных уравнений (12) почти келеровой структуры на подмногообразие $N$. С учетом (42) имеем

$$
\begin{aligned}
d \theta_{b}^{a}- & 2 d B^{c a b} \wedge \omega_{c}-2 B^{c a b} d \omega_{c} \\
= & \left(\theta_{c}^{a}-2 B^{h a c} \omega_{h}\right) \wedge\left(\theta_{b}^{c}-2 B^{r c b} \omega_{r}\right)+\left(2 B^{a d h} B_{h b c}+A_{b c}^{a d}\right) \omega^{c} \wedge \omega_{d} \\
& +B_{b c d} \omega^{c} \wedge \omega^{d}-B^{a c d}{ }_{b} \omega_{c} \wedge \omega_{d} .
\end{aligned}
$$

Подставляя сюда $\left(9_{2}\right)$ и $(14)$, с учетом (37) после необходимых сокращений получим

$$
\begin{aligned}
d \theta_{b}^{a}= & \theta_{c}^{a} \wedge \theta_{b}^{c}+\left\{\left(B^{h a g} B_{r b g}-B^{r a g} B_{h b g}\right)\right. \\
& +\frac{1}{4}\left(2 B^{a r g} B_{g b h}-2 B^{a h g} B_{g b r}+A_{b h}^{a r}-A_{b r}^{a h}\right) \\
& \left.+\frac{1}{2}\left(B_{b h r}{ }^{a}-B_{b}^{a h r}+B_{h}^{r a b}-B_{r}^{h a b}+B^{r a b h}-B^{h a b r}\right)+B^{c a b} B_{c h r}\right\} \theta^{h} \wedge \theta^{r} .
\end{aligned}
$$

С другой стороны, с учетом $(27),(32)$ и вполне геодезичности подмногообразия

$$
d \theta_{b}^{a}=\theta_{c}^{a} \wedge \theta_{b}^{c}+\frac{1}{2} \widetilde{R}_{b h r}^{a} \theta^{h} \wedge \theta^{r}
$$

Следовательно, в ортонормированном репере подмногообразия $N$

$$
\begin{aligned}
\widetilde{R}_{b c d}^{a}= & 2 B^{c a g} B_{d b g}-2 B^{d a g} B_{c b g}+\frac{1}{2}\left(2 B^{a d g} B_{g b c}-2 B^{a c g} B_{g b d}+A_{b c}^{a d}-A_{b d}^{a c}\right) \\
& +B_{b c d}{ }^{a}-B_{b}^{a c d}{ }_{b}+B_{c}^{d a b}-B_{d}^{c a b}+B^{d a b c}-B^{c a b d}+2 B^{h a b} B_{h c d} .
\end{aligned}
$$


Пусть, в частности, $M^{2 n}$ - почти эрмитово многообразие точечно постоянной голоморфной секционной кривизны $c$. Тогда, подставляя (21) в (43), после упрощений с учетом предложения 1 получим

$$
\begin{aligned}
\widetilde{R}_{b f d}^{a}= & -\frac{c}{4}\left(\delta_{f}^{a} \delta_{b}^{d}-\delta_{d}^{a} \delta_{b}^{f}\right)+B^{h a f} B_{h b d}-B^{h a d} B_{h b f}+2 B^{h a b} B_{h f d} \\
& +B_{b f d}{ }^{a}-B^{a f d}{ }_{b}+B_{f}^{d a b}-B_{d}^{f a b}+B^{d a b f}-B^{f a b d}
\end{aligned}
$$

В частности, пусть $M$ - комплексная пространственная форма голоморфной секционной кривизны $c$. Тогда в силу предложения 1 и соотношений (14) получаем, что

$$
B^{a b f}=B_{d}^{a b f}=B^{a b f d}=B_{a b f}=B_{a b f}{ }^{d}=B_{a b f d}=0,
$$

и, значит,

$$
\widetilde{R}_{b f d}^{a}=-\frac{c}{4}\left(\delta_{f}^{a} \delta_{b}^{d}-\delta_{d}^{a} \delta_{b}^{f}\right)
$$

т.е. $N$ - пространство постоянной кривизны $c / 4$. Доказана следующая теорема.

ТЕОремА 4. s-лагранжевы подмногообразия комплексной пространственной формы голоморфной секционной кривизны с являются пространствами постоянной кривизны $c / 4$.

Хорошо известно [3], что на нечетномерном многообразии $M^{2 n+1}$ аналогом симплектической структуры является контактная структура, т.е. дифференциальная 1-форма $\eta$ ранга $2 n+1$. Эта форма называется также контактной формой, а гиперраспределение ker $\eta$ - контактным распределением. Как и для симплектических структур, доказьвается, что всякая контактная структура (неоднозначно) достраивается до контактной метрической структуры, т.е. совокупности $(\xi, \eta, \Phi, g)$, где $\xi$ - вектор, $\Phi-$ тензор типа $(1,1)$ (структурньй эндоморфизм), $g=\langle\cdot, \cdot\rangle$ - риманова метрика, причем
1) $\eta(\xi)=1$
2) $\Phi(\xi)=0$,
3) $\eta \circ \Phi=0$,
4) $d \eta(X, Y)=\Omega(X, Y)$
5) $\Phi^{2}=-\mathrm{id}+\eta \otimes \xi$,
6) $\langle\Phi X, \Phi Y\rangle=\langle X, Y\rangle-\eta(X) \eta(Y)$,

где $X, Y \in \mathrm{X}(M), \Omega(X, Y)=\langle X, \Phi Y\rangle$ - 2-форма на $M$, назьваемая фундаментальной формой структуры. Такую структуру естественно назвать метрическим расширением исходной контактной структуры. Если $\eta$ - форма Киллинга, то контактная метрическая структура назьвается $K$-контактной [6]. Хорошо известно также [3], что контактное распределение допускает $n$-мерные интегральные многообразия, называемые подмногообразиями Лежандра, и не допускает интегральных многообразий большей размерности. Эти подмногообразия являются естественньг аналогом подмногообразий Лагранжа в симплектической геометрии. В частности, такими же рассуждениями, как и для подмногообразий Лагранжа, доказьвается, что через каждую точку $p$ контактного многообразия $M^{2 n+1}$ в направлении любого подпространства Лежандра (т.е. $n$-мерного подпространства, аннулирующего контактную форму и ее внешний дифференциал) $L \subset T_{p}(M)$ проходит подмногообразие Лежандра (это свойство мы назьваем полуинтегрируемостью контактного распределения). Как и в случае симплектических многообразий, доказывается, что подмногообразие Лежандра с начальньми данными $(p, L)$, вообще говоря, не единственно, однако если $\operatorname{dim} M>5$, 
то совокупность таких подмногообразий при любьх начальных данных в случае, если метрическое расширение исходной контактной структуры является $K$-контактной структурой, содержит единственное вполне геодезическое подмногообразие (это свойство мы назьваем геодезической интегрируемостью контактного распределения) тогда и только тогда, когда это расширение является сасакиевой структурой постоянной $\Phi$-голоморфной секционной кривизны (т.е. постоянной кривизны в двумерных направлениях вида $X \wedge \Phi X, X \in \operatorname{ker} \eta)$. Заметим, что многообразие, наделенное такой структурой, назьвается сасакиевой пространственной формой [13]. Легко видеть, что контактное распределение любой сасакиевой пространственной формы геодезически интегрируемо.

Вполне геодезические подмногообразия Лежандра мы назьваем подмногообразиями Блэра. Подмногообразия Блэра являются, таким образом, контактным аналогом $s$-лагранжевых подмногообразий симплектического многообразия. Как и для $s$-лагранжевых подмногообразий, проверяется, что подмногообразия Блэра сасакиевой пространственной формы $\Phi$-голоморфной секционной кривизны $c$ являются пространствами постоянной кривизны $(c+3) / 4$ (этот факт из других соображений был получен в [6]).

Укажем на одну замечательную связь между этими видами подмногообразий. Пусть $M^{2 n}$ - симплектическое многообразие с целочисленной структурной формой $\Omega$. Класс когомологий этой формы является характеристическим классом главного $T^{1}$-расслоения $\pi: P \rightarrow M$ над $M$ (расслоения Бутби-Вана [6]). Это главное расслоение допускает каноническую связность с формой $\omega$ такой, что

$$
d \omega=\pi^{*} \Omega
$$

(см. [14]). Очевидно, форма $\omega$ является контактной структурой на многообразии $P$, которую мы назовем канонической или горизонтальным поднятием исходной симплектической структуры. В силу только что сказанного имеем следующую теорему.

ТЕОРема 5. Горизонтальное распределение канонической связности в расслоении Бутби-Вана полуинтегрируемо.

Естественно называть интегральные подмногообразия горизонтального распределения горизонтальными подмногообразиями. Эти подмногообразия есть не что иное, как подмногообразия Лежандра относительно канонической контактной структуры на многообразии $P$. Из (술 немедленно следует, что проекция $N=\pi(\widetilde{N})$ горизонтального подмногообразия $\widetilde{N}$ на базу расслоения Бутби-Вана является подмногообразием Лагранжа исходного симплектического многообразия. В таком случае будем называть подмногообразие Лежандра $\widetilde{N}$ горизонтальным поднятиемподмногообразия Лагранжа $N$.

Эрмитово расширение исходной симплектической структуры многообразия $M$ мы назовем обобщенной структурой Ходжа. Оно внутренним образом порождает контактную метрическую структуру на пространстве $P$ расслоения (назовем ее горизонтальным поднятием обобщенной структуры Ходэса), контактная форма которой совпадает с формой $\omega$, контактное распределение - с горизонтальным распределением канонической связности, а фундаментальная форма - с формой $\pi^{*} \Omega$. Более того, легко видеть, что производная Ли индуцированного на $P$ метрического тензора в направлении вектора, дуального контактной форме, равна нулю, т.е. контактная форма 
является формой Киллинга, а индуцированная на $P$ контактная метрическая структура - $K$-контактной структурой [6]. Нетрудно проверить, что обобщенное многообразие Ходжа $M$ будет комплексной пространственной формой голоморфной секционной кривизны $c$ тогда и только тогда, когда пространство расслоения Бутби-Вана над ним будет сасакиевой пространственной формой $\Phi$-голоморфной секционной кривизны $c-3$. С учетом сделанных замечаний получаем следующее утверждение.

ТЕорема 6. Горизонтальное распределение канонической связности в расслоении Бутби-Вана над обобщенным многообразием Ходжа М размерности свыше четырех геодезически интегрируемо тогда и только тогда, когда $M$ - комплексная пространственная форма.

Пусть $N \subset P$ - подмногообразие Блэра. Из определения метрики, индуцированной в пространстве расслоения Бутби-Вана [6], с учетом рассуждений из доказательства теоремы 1, вполне идентичных как в симплектическом, так и в контактном случаях, убеждаемся, что, во-первых, сужение естественной проекции $\pi$ на подмногообразие Лежандра является (локальной) изометрией; и, во-вторых, это отображение вполне геодезические подмногообразия Лежандра переводит во вполне геодезические подмногообразия Лагранжа, т.е. подмногообразия Блэра - в $s$-лагранжевы подмногообразия. Таким образом, подмногообразия Блэра являются горизонтальньми поднятиями $s$-лагранжевых подмногообразий. Более того, из определения горизонтального поднятия обобщенной структуры Ходжа [6] следует, что соответствующая $K$-контактная структура инвариантна относительно действия структурной группы главного $T^{1}$-расслоения, которое, таким образом, подмногообразия Лежандра переводит в подмногообразия Лежандра, а подмногообразия Блэра - в подмногообразия Блэра. Пусть $N \subset M-$ $s$-лагранжево подмногообразие комплексной пространственной формы, $p \in N$ - произвольная точка, $q \in \pi^{-1}(p), L \subset T_{q}(P)$ - горизонтальный подьем пространства $T_{p}(N)$, $\mathscr{P} \subset P$ - максимальноеподмногообразие Блэра с начальными данными $(q, L)$. Рассмотрим подгруппу $G$ структурной групшы $T^{1}$ расслоения Бутби-Вана, переводящую подмногообразие $\mathscr{P}$ в себя (т.е. группу инвариантности этого подмногообразия). Очевидно, это собственная и, значит, дискретная подгруппа. В силу компактности $T^{1}$ эта подгруппа конечна. Орбита каждой точки из $\mathscr{P}$ относительно нее есть не что иное, как пересечение полного прообраза проекции этой точки на базу с $\mathscr{P}$. Пусть $U$-окрестность точки $q$ в $\mathscr{P}$, сужение на которую отображения $\pi$ является изометрией. Поскольку $\pi$ - открытое отображение, подмногообразие $\widetilde{N}=\pi(\mathscr{P}) \subset M$ является $s$-лагранжевьм подмногообразием с начальными данными $\left(p, T_{p}(N)\right)$, определенньм в окрестности $\pi(U)$ точки $p$. В силу теоремы 3 это подмногообразие совпадает с $N$ в некоторой окрестности $W$ точки $p$. Рассмотрим $\pi^{-1}(W) \cap \mathscr{P}$. В соответствии со сказанным

$$
\pi^{-1}(W) \cap \mathscr{P}=\bigcup_{i=1}^{n} W_{i},
$$

где $n$ - число элементов подгрупшы $G$, причем $W_{i}$ переводится в $W_{j}$ действием соответствующей изометрии из $G$, а $\pi\left(W_{i}\right)=W(i, j=1, \ldots, n)$. Ввиду произвола $p \in N$ отображение $\pi: P \rightarrow M$ индуцирует накрытие $\varpi: \mathscr{N} \rightarrow N$, где $\mathscr{N}=\pi^{-1}(N) \cap \mathscr{P}-$ подмногообразие Блэра, которое мы назовем полным горизонтальным поднятием $s$-лагранжева подмногообразия $N, \varpi=\pi \mid \mathcal{N}$. Таким образом, доказана 
ТЕОРема 7. Каждое $s$-лагранжево подмногообразие $N$ комплексной пространственной формы $M$ конечнолистно накрывается некоторым подмногообразием Бләра в расслоении Бутби-Вана над $M$, являющимся полным горизонтальным поднятием подмногообразия $N$. При этом группа инвариантности подмногообразия Бләра является подгруппой фундаментальной группы соответствующего $s$-лагранжева подмногообразия.

СледСтвИЕ. Каждое односвязное $s$-лагранжево подмногообразие $N$ комплексной пространственной формы $M$ изометрично некоторому подмногообразию Бләра с тривиальной группой инвариантности в расслоении Бутби-Вана над $M$, являюшемуся полным горизонтальныцм поднятием подмногообразия $N$.

\section{СПИСОК ЦИТИРОВАННОЙ ЛИТЕРАТУРЫ}

[1] Фоменко А. Т. Симплектическая геометрия. Методы и приложения. М.: Изд-во МГУ, 1988.

[2] Fomenko A. T. Symplectic Geometry (2nd ed.). Adv. Stud. Contemp. Math. V. 5. Amsterdam: Gordon and Breach Publishers, 1995.

[3] Арнольд В. И. Математические основы классической механики. М: Наука, 1989.

[4] Арнольд В.И., Гивенталь А. Б. Симплектическая геометрия // Современные проблемы математики. Фундамент. направления. Т. 4. М.: ВИНИТИ, 1985. С. 7-139.

[5] Дубровин Б. А., Новиков С.П., Фоменко А. Т. Современная геометрия. Методы и приложения. 2-е изд. М.: Наука, 1986.

[6] Blair D. E. Contact Manifolds in Riemannian Geometry. Lecture Notes in Math. V. 509. Berlin-Heidelberg-New York: Springer-Verlag, 1976.

[7] Лихнерович А. Теория связностей в целом и группы голономии. М.: ИЛ, 1960.

[8] Кобаяши Ш., Номидзу К. Основы дифференциальной геометрии. М.: Наука, 1981.

[9] Gray A., Hervella L. M. The sixteen classes of almost Hermitian manifolds and their linear invariants // Ann. Math. Pure Appl. 1980. V. 123. № 4. P. 35-58.

[10] Кириченко В. Ф. Методы обобщенной эрмитовой геометрии в теории почти контактных многообразий // Итоги науки и техн. Проблемы геометрии. Т. 18. М.: ВИНИТИ АН СССР, 1986. C. $25-71$.

[11] Kirichenko V.F. Generalized quasi-Kaehlerian manifolds and axioms of $C R$-submanifolds in generalized Hermitian geometry. II // Geometriae Dedicata. 1994. V. 52. P. 53-85.

[12] Kirichenko V.F. Generalized quasi-Kaehlerian manifolds and axioms of $C R$-submanifolds in generalized Hermitian geometry. I // Geometriae Dedicata. 1994. V. 51. P. 75-104.

[13] Ishihara I. Anti-invariant submanifolds of a Sasakian space form // Kodai Math. J. 1979. V. 2. P. $171-186$.

[14] Kobayashi S. Principal fibre bundle with the 1-dimensional toroidal group // Tôhoku Math. J. 1956. V. 8. P. 29-45. 